\title{
SIGNAL MEASUREMENT SYSTEM DESIGN BIOMECHANICS OF THE HANDS FOR ASSESSMENT IN PATIENTS WITH PARKINSON
}

\section{JACOB ASTOCONDOR, JUAN GRADOS, WILVER AUCCAHUASI, SANTIAGO RUBIÑOS, CÉSAR SANTOS, JESSICA MEZA, ABILIO CUZCANO}

Facultad de Ingenieria Electrica y Electronica, Universidad Nacional del Callao, Lima, Perú

\begin{abstract}
The present research work offers an application for the measurement of the biomechanical signals of the hand in patients with Parkinson's disease. To analyze these movements and their subsequent evaluation, techniques based on inertial sensors are used. In the present development, a dsPIC of the dsPIC33F family was considered in the processing stage. We worked with the opening and closing of the hand, pronation and supination movements, the signals produced by these movements were recorded, then it was analyzed and the results show that it can be considered as an aid in the medical diagnosis, both for the specialties of physical medicine such as traumatology, for the treatment of Parkinson's, the specialty of neurology becomes vital because its evaluation has the characteristic of being subjective, which generates in some cases, diagnostic problems due to the weak precision of the evaluation and these lead to a deficient treatment, with the advancement of technology there are now sensors and processors specialized in signal measurement systems which can help to improve the precision in the diagnosis, in this work we present a mechanism for measuring the movement of the hands which allows us to measure the degree of frequency and amplitude of the movements to improve the diagnosis of Parkinson's.
\end{abstract}

KEYWORDS: Parkinson's, Inertial Sensor, UPDRS Scale, Pronation, Supination

Received: Jun 08, 2020; Accepted: Jun 28, 2020; Published: Sep 05, 2020; Paper Id.: IJMPERDJUN20201078

\section{INTRODUCTION}

The analysis of human movement is an area of research in biomechanics where it is sought to explain, using various types of sensors, what movements a person performs and how they are performed [1]. An important task in this area of research lies in proposing computational models capable of representing human movement [2]. The measurement of hand movement is important in many fields, such as functional evaluation in pathological diagnosis, follow-up in rehabilitation, analysis of sports technique or ergonomic evaluation of product use [3].

Different techniques such as goniometers, instrumented gloves or motion capture from images are used for its measurement [4]. Many times, most, it is desirable to measure movement through the use of techniques that do not interfere with the normal development of the activities to be performed by hand [5]. In the analysis of movements, various devices are used to record movement, such as those found in video games [6], gyroscopes are the most widely used mechanism due to the ease of recording and the analysis provided by measuring each of the axles [7].

In this research work, the design of a system for measuring data on the tremor of the hands of patients with Parkinson's is carried out, using inertial accelerometer sensors and processor (micro controller), the data will be stored in memory, to be later analyzed and later make the assessment of the signals and their treatment. 


\section{METHODS \& MATERIALS}

\section{Biomechanics and Hand Movement}

Biomechanics studies the analysis of the mechanics of movement of the human body. It is the science that explains how and why the human body moves the way it does. This includes the interaction between the person executing the movement and the equipment or environment.

In biomechanics, it considers the concepts of kinetics (study of forces acting on the body) and kinematics (study of body movements). The most important components of biomechanics are indicated as: movement, force, moment, levers and balance:

\section{Movement}

It is the movement of the body or an object through space. Speed and acceleration are important components of motion.

\section{Fuerza}

It is the push or pull that causes a person or object to speed up, slow down, stop, or change direction.

\section{Moment}

It is the result of a mass and its speed in its displacement.

\section{Levers}

The upper and lower extremities (arms and legs) work like levers; A lever is made up of three components: the resistance arm, the fulcrum and the axis of rotation.

\section{Balance}

An important principle of balance is the alignment of the body's center of gravity on the base of support. Good balance is important for many sports and exercises.

\section{Characteristics of Parkinson}

Parkinson's, considered as a clinical picture consisting of slow movement (bradykinesia), together with tremor at rest and increased muscle tone (rigidity). The name Parkinsonian Syndrome describes the set of signs and symptoms (this is what syndrome means) characterized by rigidity, tremor and motor clumsiness, when this is due to other neurological causes.

Poor movements, clumsiness, slowness, Decreased facial movements with poor expressiveness, reduction in spontaneous blinking and stroking during walking, slow dressing or grooming, loss of ability and dexterity, writing disorders that become slow and small lines, difficulty chewing and swallowing. Decreased tone of voice. Loss of intonation when speaking. The march becomes slower with difficulties in turning. In some cases, irrepressible acceleration when walking, with difficulty standing.

Tremor, At first it is interpreted as nervousness. It is usually a tremor at rest that, in the early stages, decreases when a movement begins. It affects the arms, and later the legs.

Rigidity, Increased muscle tone. Usually the patient does not appreciate it, but the doctor discovers it easily when he tries to passively move his limbs. 


\section{Inertial Measurement Sensors}

The sensor called inertial measurement unit (Inertial Measurment Units IMU) allows us to measure the orientation of a body, it combines an accelerometer with axes and a gyroscope.

IMU sensors are widely used in navigation, goniometry, stabilization, etc. These sensors have accelerometers, gyroscope, magnetometer, barometer, there is also internally a processor with communications protocol.

\section{Medicion Con El Acelerometro}

The accelerometer measures acceleration in 3 axes: $\mathrm{X}, \mathrm{Y}$ and $\mathrm{Z}$, the three dimensions of space. For example, if you move up, the $\mathrm{Z}$ axis will mark a certain value. If it is forward, it would mark the $\mathrm{X}$ axis, etc.

As we know that the Earth's gravity has an acceleration of approx. $9.8 \mathrm{M} \mathrm{S} 2$, perpendicular to the ground. Thus, the IMU also detects the acceleration of Earth's gravity. Thanks to the earth's gravity, the accelerometer readings will be made; to determine what is the angle of inclination with respect to the $\mathrm{X}$ axis or $\mathrm{Y}$ axis.

\section{Gyroscope and Angular Velocity}

Angular velocity is the amount of change in angular displacement per unit of time, that is, how fast a body rotates around its axis: Gyroscopes use a MEMS (MicroElectroMechanical Systems) to measure angular velocity using the Coriolis effect With a gyroscope we measure the angular velocity, and if the angular velocity is integrated with respect to time, the angular displacement is obtained (angular position if it is known where the turn started).

\section{Motion Capture Implementation}

The movement of flexion and extension that is captured would be around the $\mathrm{Z}$ axis of the proposed coordinate system and the abduction and adduction in the axis around the $\mathrm{Y}$ axis. The coordinate system is located at the wrist of the hand and from this the sensor towards the middle of the hand where it is positioned due to the use of an accessory to carry it comfortably.

The motion capture was made through the MPU6050 inertial sensor, this was chosen because it allows integrating information from the accelerometer and the gyroscope by merging data, which has the advantages; reduce uncertainty and increase the precision of the census values, increase the reliability in the event that one of the sensors fails, and collect complementary information from the environment being characterized, which cannot be obtained with a single sensor; all this with the aim of obtaining a more adequate data record. Data transmission is done through the I2C protocol to the micro controller (dsPIC). The pins for the I2C protocol layout on the dsPIC4013 SDA and SCL One are Pin 26 and Pin 25 which correspond to SDA (Data Line) and SCL (Clock Line) respectively; on the MPU6050 sensor they are found on the third and fourth pins in the same order.

The correct way to connect the two devices is: polarize the MPU6050, the voltage is already regulated for $5 \mathrm{~V}$, so there is no problem in connecting directly, connecting the grounds, connecting the SDA of the dsPIC with the SDA of the sensor, as well as the SCL of the sensor with the SCL of the dsPIC, finally the program is made so that every time the sensor detects an interruption signal I send the data through the SDA, therefore it is necessary to connect the INT pin of the sensor, which is the 'Lastly, to pin 17 of the dsPIC, which is the one that is arranged in the dsPIC to send the signal. 


\section{Considerations}

In MARG sensors (Magnetic, Angular Velocity, Gravity), each of the sensors of which they are composed presents some advantages and disadvantages in the measurements; such as drift errors, sensitivity to mechanical noise or magnetic inferences.

The objective of data fusion algorithms is to reduce the deficit of a sensor, with the complementary information of the other sensors, providing more precise information on the orientation of the device.

The accelerometer and magnetometer give us coordinates of a vector in the sensor system, since we know the orientation of these vectors in the earth system, which are, gravity and the earth's magnetic field, we can calculate the orientation of the sensor system with respect to to the earth system, with the disadvantage that there are infinite orientations that the sensor can have, with coordinates equal to those of the vector, which are the rotations of the system with the vector as the axis of rotation, for which the Madgwick algorithm is implemented.

\section{RESULTS}

\section{Sensor Selection}

The MPU-6050 was chosen as the sensor element of the project, especially considering the fact that it combines an accelerometer and a gyroscope in the same device. The MPU-6050 combines a 3-axis gyroscope and a 3-axis accelerometer on the same chip. It also incorporates a motion processor capable of processing a complex 9-axis motion fusion. It communicates through the $\mathrm{I} 2 \mathrm{C}$ interface and has a very popular library for immediate use. This sensor can deliver 6 degrees of freedom (DOF) and incorporates a 3.3V voltage regulator and pull-up resistors for direct use by I2C.

Using the i2cdevlib library facilitates the use to carry out the project where it is required to control the inclination, rotation and altitude.

\section{IMU MPU6050 Specifications}

- Supply voltage: $3-5 \mathrm{v}$

- Digital interface: I2C Digital

- 3-axis angle sensor (gyroscope)

- 3 -axis accelerometer with programmable precision in ranges of $\pm 2 \mathrm{~g}, \pm 4 \mathrm{~g}, \pm 8 \mathrm{~g}, \pm 16 \mathrm{~g}$

- Embedded algorithms to improve calibration without user intervention.

- Dimensions: $14 \times 21 \mathrm{~mm}$

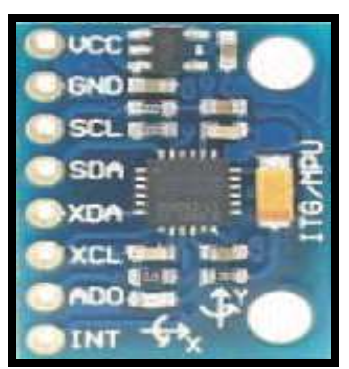

Figure 1: Sensor View. 


\section{Gyroscope Sensor}

A gyroscope is a device that works to measure angular velocities based on maintaining the momentum of rotation. If we tried to rotate an object that is rotating about an axis that is not the axis about which it is rotating, the object would exert a moment opposite to the movement in order to preserve the total rotational momentum. The gyroscope shows the range change in rotation on its $\mathrm{X}, \mathrm{Y}$ axes.

\section{Sensor Location for Data Collection}

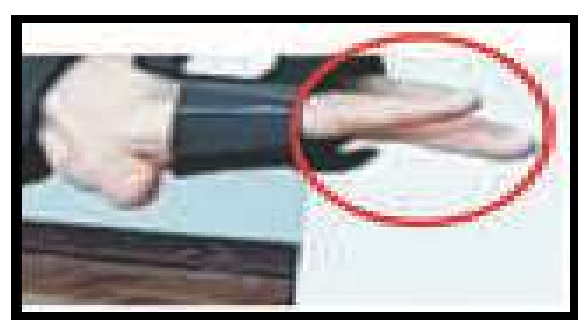

Figure 2: Sensor Location.

The system is based on sensors, placed in the hand, which is in charge of detecting the typical tremors of Parkinson's disease and sends the captured data to a computer through a microcontroller, the system has the possibility of sending data via Bluetooth 2.0 to another terminal with a Bluetooth receiver so that the signals can be viewed. The system is ready to process data online due to a dsPIC33F microcontroller capable of controlling all processes and performing the necessary calculations to be able to treat, process and execute algorithms.

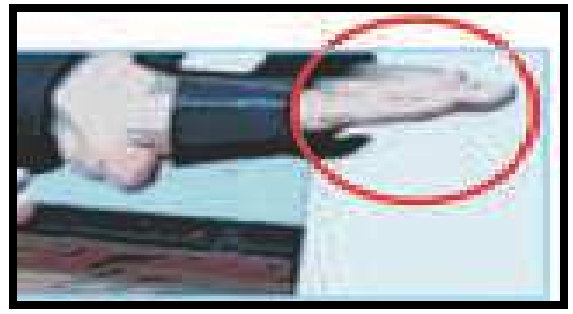

Figure 3: Sensor Location.

\section{Obtaining Speed}

After obtaining the signal with the sensors, it was then gravitationally compensated, in order to have the earth as a reference and obtain the completely clean acceleration for its treatment. It is indicated that the position, velocity, acceleration, are expressed in terms of the derivatives and integrals Mathematical position relations.

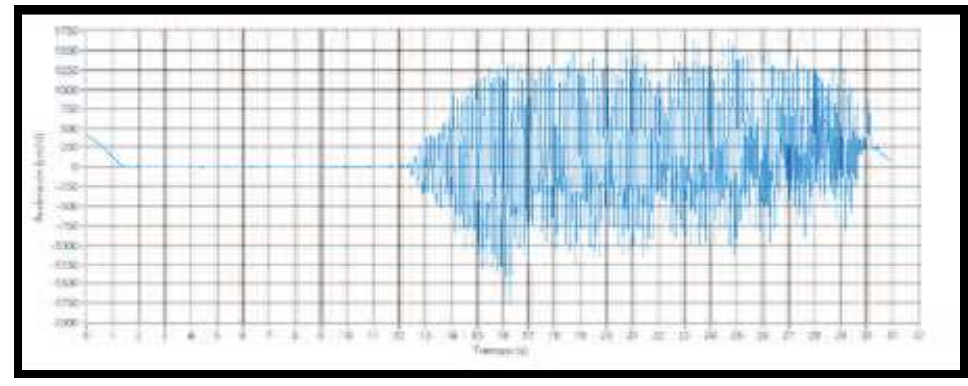

Figure 4: Acceleration Signal Compensated. 


\section{Speed Conditioning}

Integrating the acceleration signal the speed is obtained, performing the algorithm, the graph is obtained. A displacement in the vertical axis is observed, and then the speed is stable with very small variations and finally an abrupt change due to the movement of the patient's hand due to the exposure time they were in.

To improve the precision of the sampling, it is necessary to consider a high pass filter to eliminate noise and signals that do not correspond to the Parkinsonian signal. The filter removes unwanted signals and also cleans the signal to be considered for treatment.

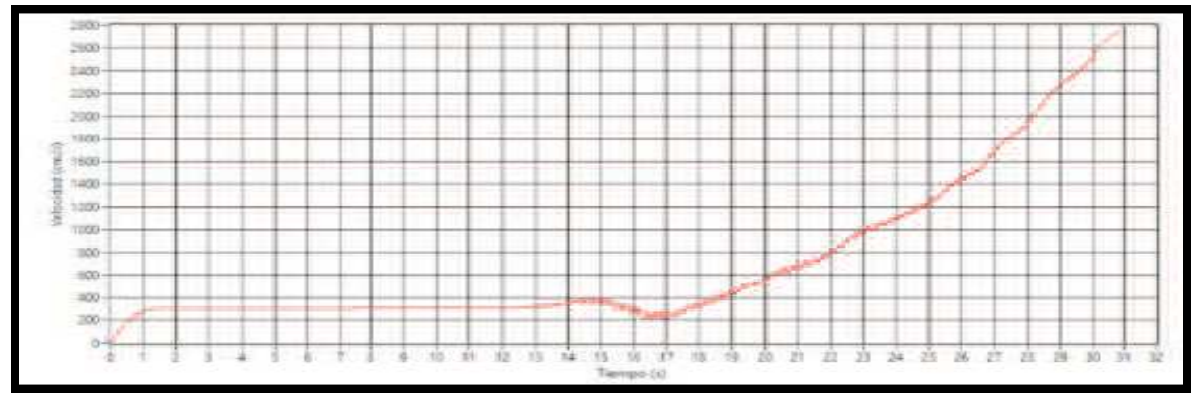

Figure 5: Speed of Hand Tremors.

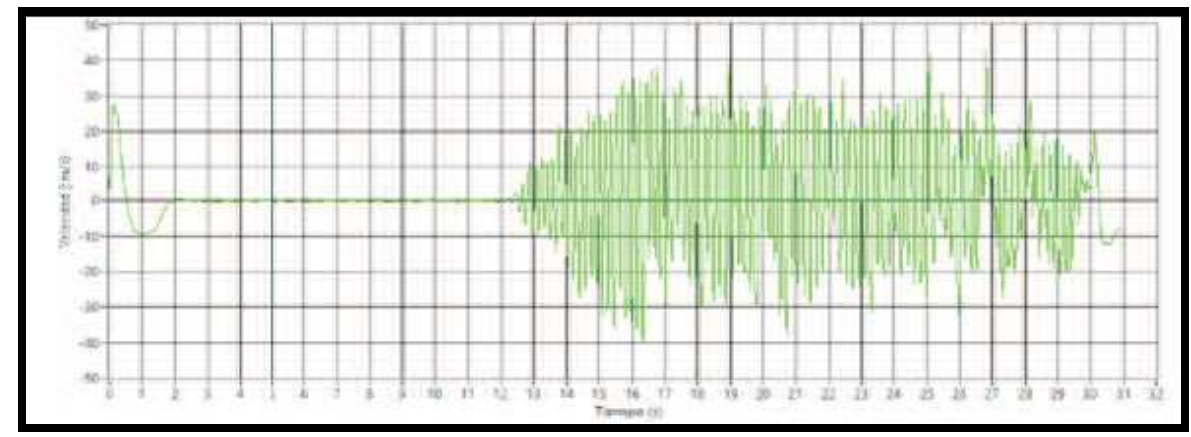

Figure 6: Speed of Hand Tremors, Filtered with Noise.

\section{Obtaining Hand Positioning}

After filtering the velocity of the parkinsonian movement of the hand, the

It performs the mathematical process integrating the speed to obtain the data of the hand positioning, then the filtering process is carried out to clean the signal and eliminate the parasitic signals, which does not correspond to the Parkinsonian process.

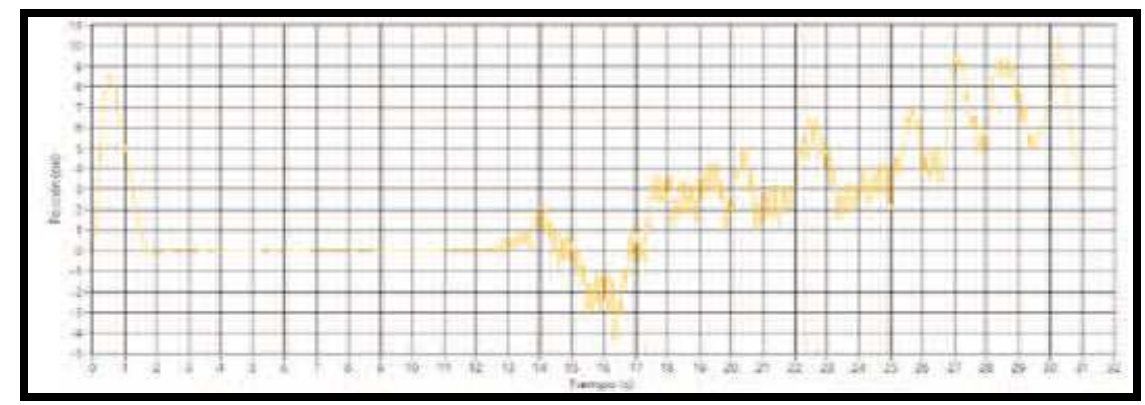

Figure 7: Speed of Hand Tremors, Filtered Without Noise. 
Then a high pass filter with a cutoff frequency of $1.2 \mathrm{~Hz}$ is considered, to eliminate the signals with very low frequencies that correspond to noise, obtaining the positioning data of the filtered hand.

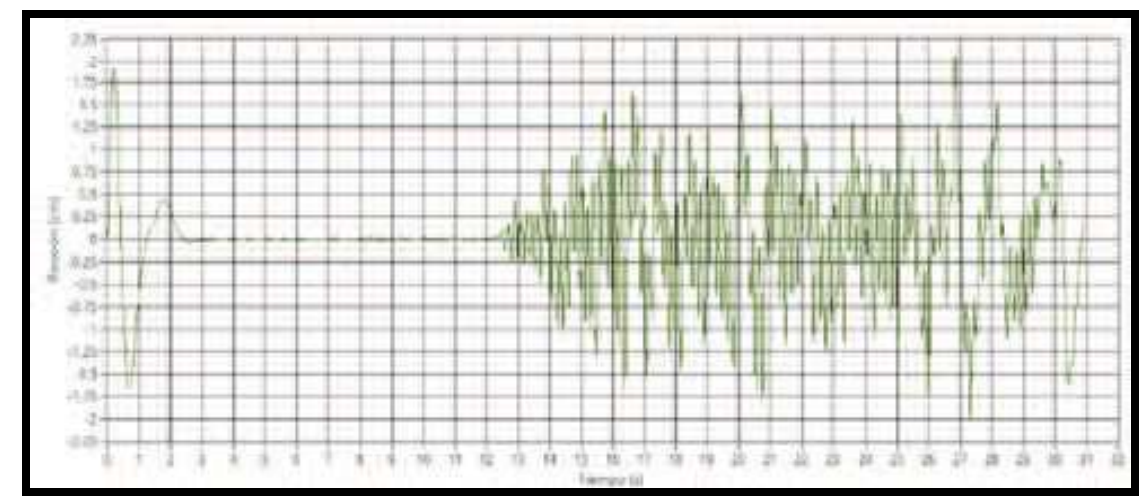

Figure 8: Hand Position with Tremors with Filtered Noise Data.

\section{Measurement of Maximum Values of the Position Measured Signal}

To determine the maximum and minimum values of the data, it was carried out with the software Matlab. Using the data and the findpeaks function to find values and locations of

Local maxima in a data set. The data.mat file contains the average number of data observed during a time sampled from the patient's hand signal.

The inverse of data is entered.m; to obtain the local minimum in the displacements.

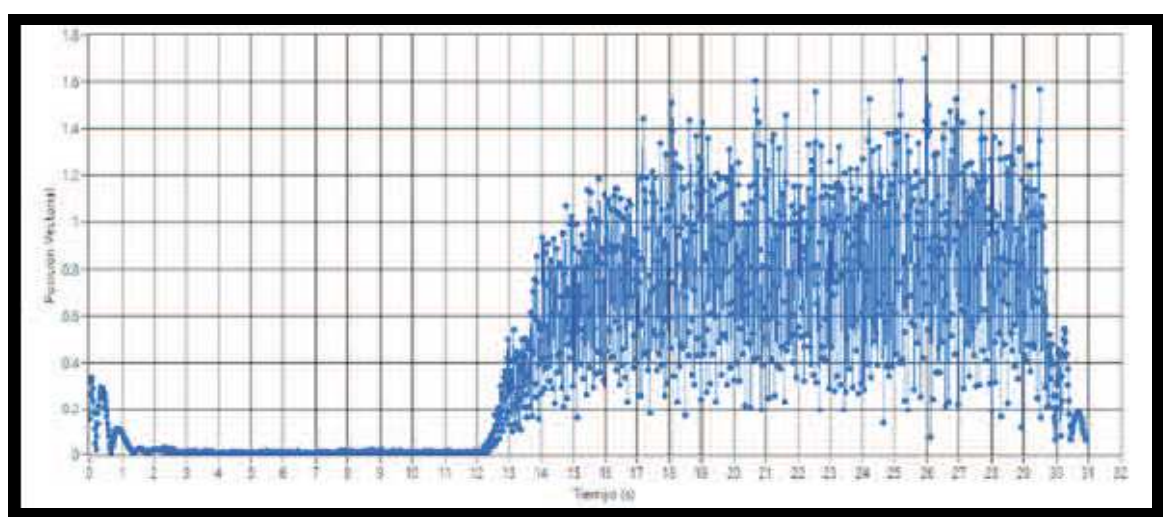

Figure 9: Hand Position with Tremors with Data Obtained in Vector Form.

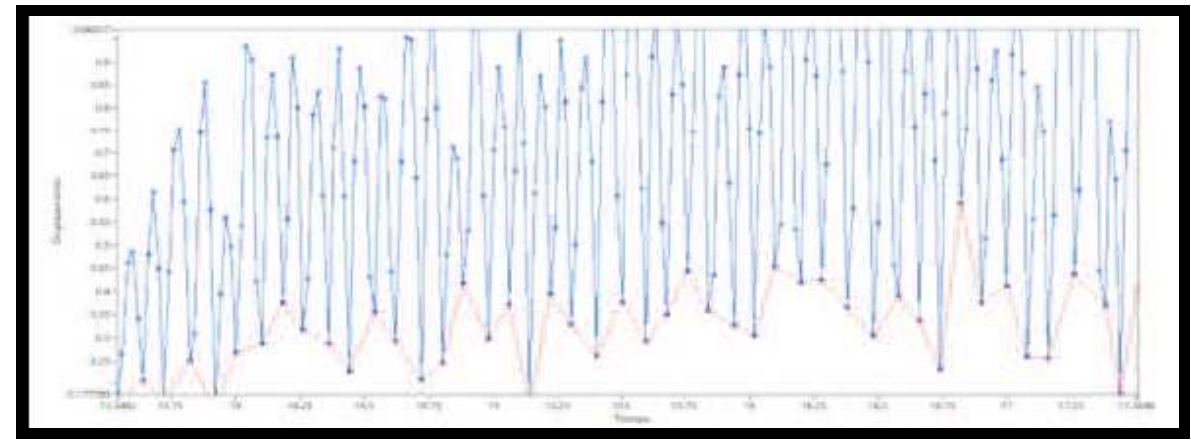

Figure 10: Displacement of the Position of the Hand with Tremors with Maximum and Minimum. 
It is indicated that the minimum and maximum level correspond to the values of the data taken is when you have the hand tremors; considering that tremors are oscillations; the sensors measure the decrease in acceleration; Because the oscillations can be considered as a round trip (return), then we can say that the speed, acceleration and displacement, increases to the point where the return or return occurs in the hand (return oscillation).

\section{Determination of the Amplitude of Oscillation of the Hand}

When the minimum calculation is performed, the points where the hand oscillates or trembles are obtained, indicating at these points is where the increase or decrease in the amplitude of the oscillation of the hand changes. Calculations were made to determine the positions (integrating and adjusting), obtaining the movements in the 3 axes of the sensor; To calculate the amplitude, the minimum levels and the time in which each vector displacement occurred were taken, between each minimum and maximum time displacements were obtained.

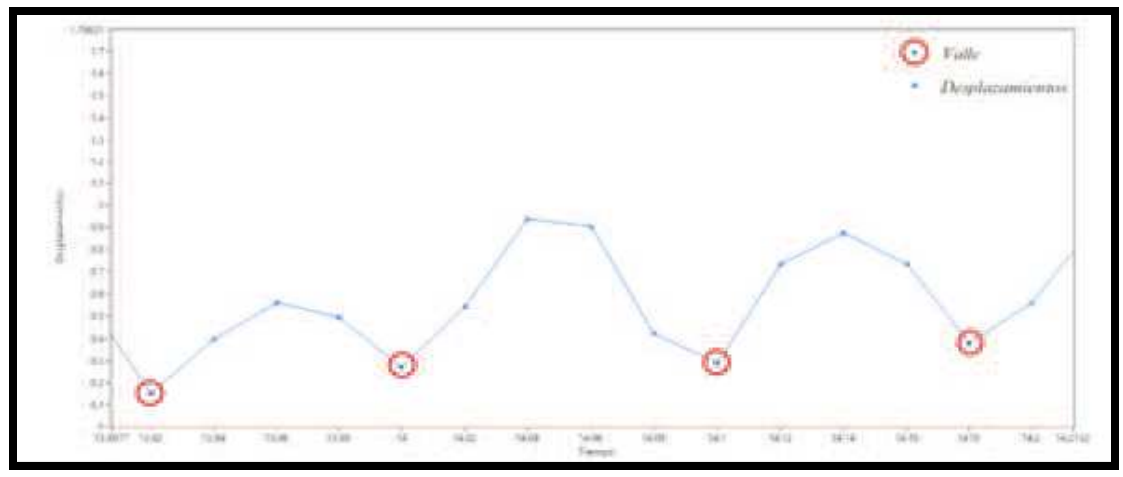

Figure 11: Minimum Value of the Hand Positions with Tremors.

The figure shows the data obtained during a Delta $\mathrm{T}$ time (Start time and end time) between each minimum value; then the calculation is performed to determine the linear amplitude between each minimum value.

\section{Most Significant Amplitude of Motion Data}

The MDS-UPDRS indicates that to assess tremors at rest, it is necessary to record the amplitude of the most significant movement.

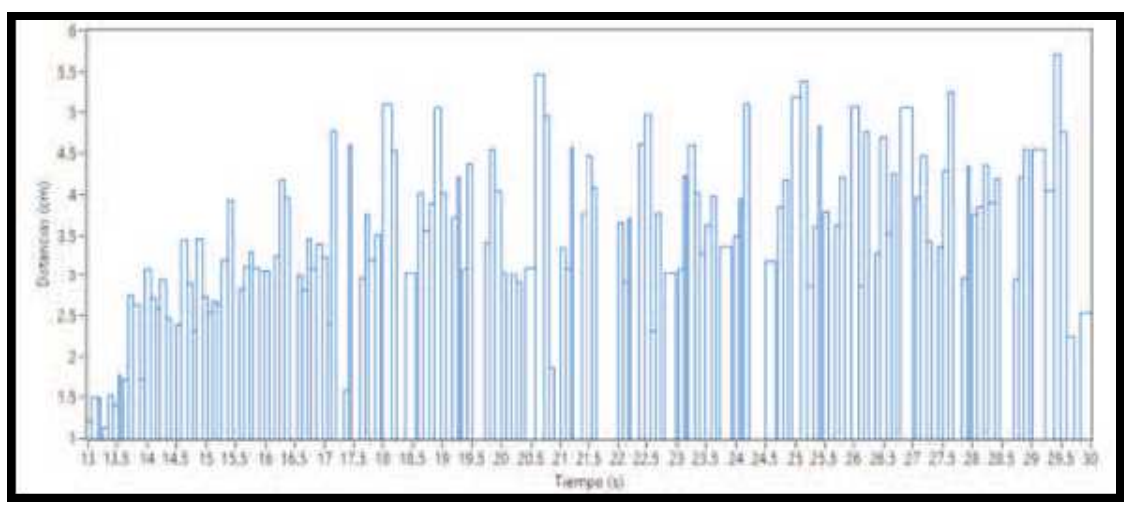

Figure 12: Distance Values of the Adjusted Amplitudes of the Hand Tremors.

When carrying out the evaluation and using the largest measured amplitude, it could not be a parkinsionan movement, then the procedure of considering an average of the data is carried out, in this case a third of the data. 


\section{Evaluation of Parkinsonian Signs}

To evaluate the Parkinsonian signals, a FUZZY control evaluation system was considered to evaluate the biomechanical indicators. The inputs to the fuzzy control system were considered:

- The amplitude of the hand shaking

- The frequency of the hand shaking

- The difference of the unforeseen values of the hand

\section{Diffuse Logic}

A theory of the Fuzzy system is carried out as a reference of the system to be developed. Fuzzy logic is applied to various areas, one of them being in measurement systems.

These intelligent controllers have the advantage of being able to stabilize non-linear systems and work in complex processes such as allowing to carry out control strategies

Used by human operators, that is, they use the rules of the human expert of the form

IF-THEN, Range of the fuzzy model variables.

\section{Entry}

- A: Amplitude: a range of $[0 ; 15]$, since the values of

- F: Frequency: a range between $[0 ; 10]$.

- D: Difference: the range between [0; two]

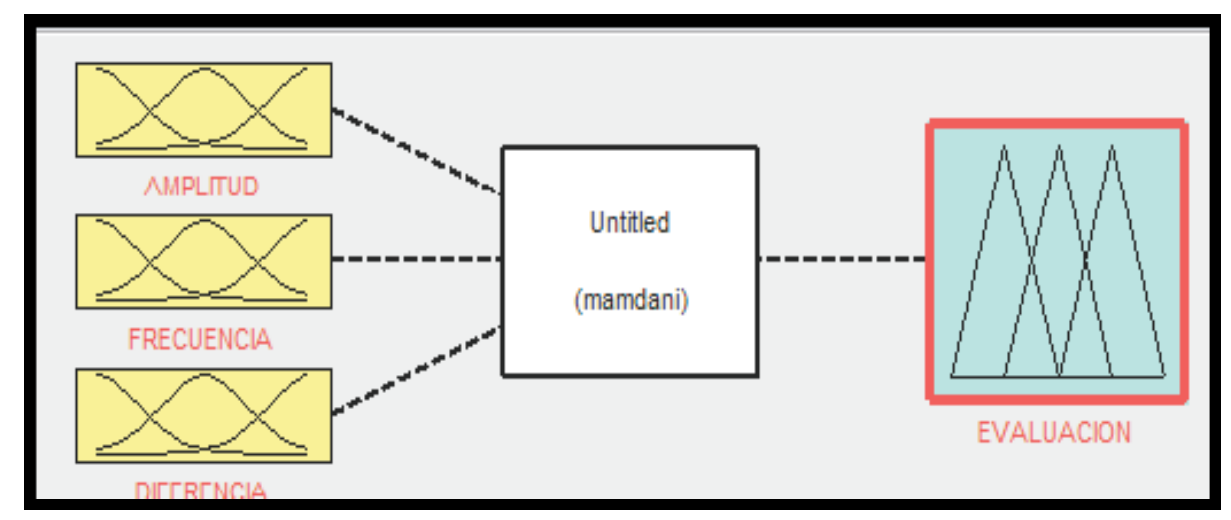

Figure 13: Architecture of the Proposed System.

Exit

- S: Output variable: A range is established between $[0 ; 4]$.

This output variable is based on the unified measurement scale for Parkinson's disease, where the established range indicates an output of 0 the patient is in a normal state and 4 is a patient with severe disease. 


\section{Membership Features}

- Amplitude membership functions (input): 5 functions have been programmed

- Membership, 4 triangular membership functions and 1 membership function

- $\quad$ Trapezoidal, this is represented in table No. 03, where the ranges are indicated

\section{Respectives}

Representation of the membership function of the amplitude in graphical form, carried out with the fuzzy program, this is represented with its range of values, it has been considered; the membership values in order to obtain an assessment considering the MDS-UPDRS scale, taking into account the amplitude of the hand tremors and assessing with the terms:

- Very Low: Equivalent to a normal patient with hand tremor without tremor

- Low: Equivalent to a patient with minimal hand tremor

- Medium: Equivalent to a patient with mild hand tremor

- High: Equivalent to a patient with moderate hand tremor

- Very high: Equivalent to a patient with severe hand tremor

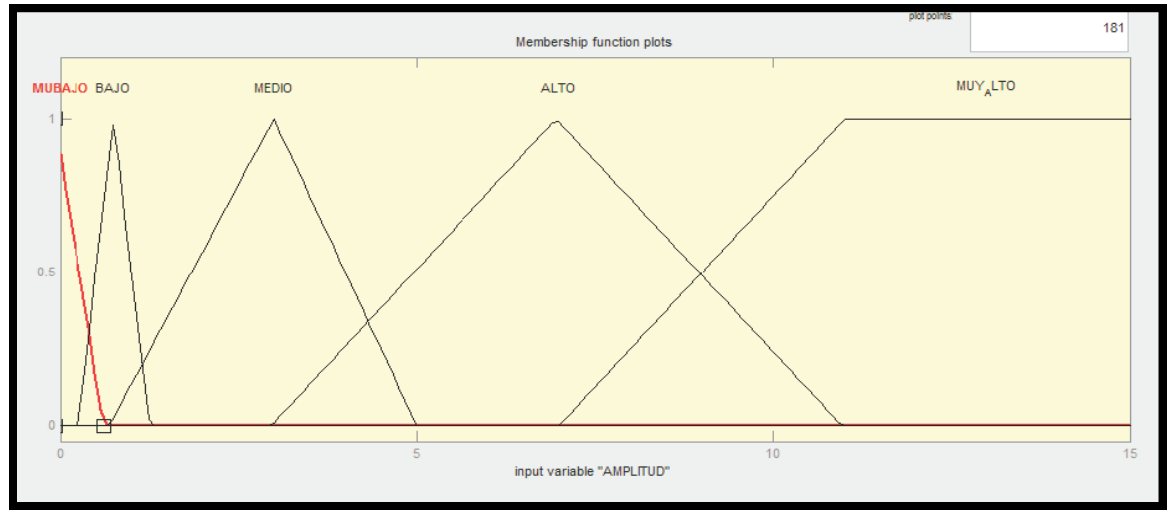

Figure 14: Membership Function of the Amplitude of the Hand Tremors for the Range of 0 To $15 \mathrm{~cm}$.

The frequency membership function is represented in graphical form, this is done with Matlab's Fuzzy software; we have considered two trapezoidal functions and one triangular with their respective ranges

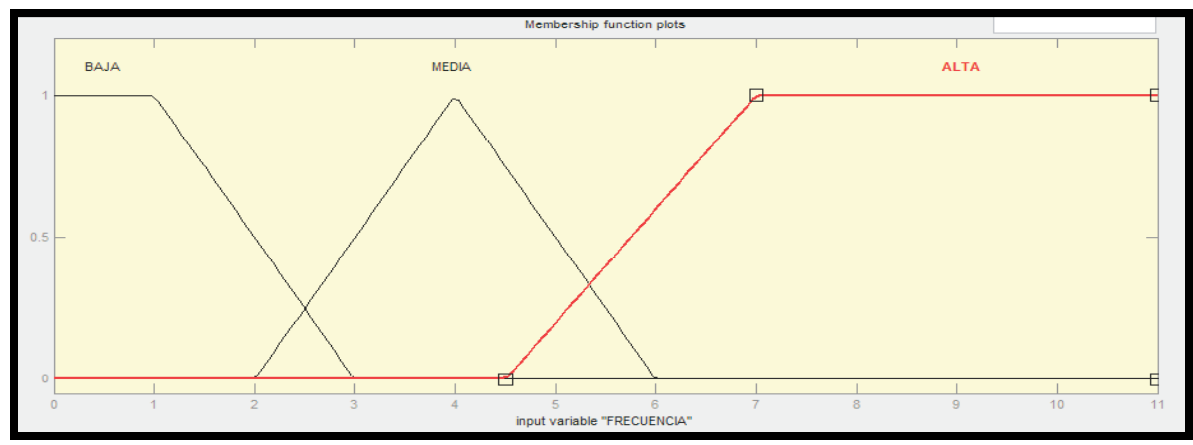

Figure 15: Frequency Membership Function. 


\section{CONCLUSIONS}

A functional system was designed for the measurement of the biomechanical signal of the human hand, using IMU sensors (inertial measurement units containing Accelerometer, gyroscope and magnetometer) a data acquisition card based on the microcontroller dsPICFJMC202 from the microchip company, then An ATMEL328P card and a USB connection cable were also used to connect to a PC, later the connection was made with a communication system with bluetooch, as a way to make wireless measurements up to 4 meters away.

The measurement system was designed to measure the amplitude and frequency of the biomechanical signals of the patient under study; The concept of double integration of the accelerometer was applied to obtain the position and frequency of the sensor in the patient's hand and the signal was cleaned with filters so as not to measure parasitic signals that correspond to the movement or tremor of the hand.

To perform the assessment of the biomechanical signals of the patient, a Fuzzy system was developed, having as input to the diffuse model the amplitude, frequency and the difference of the amplitudes of the biomechanical signals.

The MDS-UPDRS rules are considered to designate the linguistic variables of the model output:

- Normal

- Minimum

- Moderate

- Severe

For the final evaluation, the doctor's recommendations are considered and the functioning of the measurement system is verified.

\section{REFERENCES}

1. Brinckmann P, Frobin W, Leivseth G y H.D. Sherali, Musculoskeletal Biomechanics. Thieme, pags 4,16, 2002.

2. D. Brero, M. Prado, Desarrollo de un modelo biomecanico multicuerpo de la mano y aplicacion a la caracterizacíon de su movimiento con tecnicas de estereo videogrametria,CIBIM 10 p'ags, 2515-2526., 2011.

3. Dufour M, Pillu M, Biomec'anica Funcional, Miembros, cabeza, tronco,Masson,pags 351,412,2006,

4. Field D, Palastanga $N$, Field D, Soames $R$, Anatomia y movimiento humano: estructura y funcionamiento,3ra. Ed. Paidotribo,p 67, 2000.

5. M. Saucedo, Valoracion de la marcha humana, Mexico, 2009.

6. TextscJ. Raheja, A. Chaudhary y K. Singal, Tracking of Fingertips and Centers of Palm Using KINECT., Computational Intelligence, Modelling and Simulation (CIMSiM), pp. 248-252, 2011.

7. TextscR. E. Mayagoitia, A. V. Nene y P. H. Veltink, Accelerometer and rate gyroscope measurement of kinematics: an inexpensive alternative to optical motion analysis systems, Journal of biomechanics, vol. 35, no4, pp. 537-542, 2002. 
